\title{
Neutralino-Sbottom Coannihilation in SU(5)
}

\author{
Ilia Gogoladze ${ }^{1}$, Shabbar Raza ${ }^{2}$ and Qaisar Shafi ${ }^{3}$ \\ Bartol Research Institute, Department of Physics and Astronomy, \\ University of Delaware, Newark, DE 19716, USA
}

\begin{abstract}
We identify within the $S U(5)$ framework the minimum number of soft supersymmetry breaking parameters which can yield a bottom squrak (sbottom) as the next to lightest supersymmetric particle. We focus in particular on the neutralino-sbottom coannihilation scenario which gives rise to the desired neutralino dark matter relic density. We find solutions in which the sbottom mass is greater than or of order $210 \mathrm{GeV}$, while the gluino and the first two family squarks are heavier than $1 \mathrm{TeV}$. Some benchmark points which can be tested at the LHC are presented.
\end{abstract}

\footnotetext{
${ }^{1}$ Email: ilia@bartol.udel.edu. On leave of absence from: Andronikashvili Institute of Physics, GAS, Tbilisi, Georgia.

${ }^{2}$ Email: shabbar@udel.edu. On study leave from: Department of Physics, FUUAST, Islamabad, Pakistan.

${ }^{3}$ Email: shafi@bartol.udel.edu.
} 


\section{Contents}

1 Introduction 1

2 Soft Supersymmetry Breaking (SSB) Parameters in $S U(5) \quad 2$

3 Phenomenological constraints and scanning procedure 2

4 Sbottom Coannihilation 4

$\begin{array}{lll}5 & \text { Conclusions } & 11\end{array}$

\section{Introduction}

In a recent paper [1], the authors have shown that $b-\tau$ Yukawa coupling unification, realized in some well motivated $S U(5)$ and $S O(10)$ models, is compatible with the constrained minimal supersymmetric model (CMSSM) and with neutralino dark matter abundance only if there exists neutralino-stop coannihilation. In order for this coannihilation scenario to be effective, the lighter stop must be the next to lightest supersymmetric particle (NLSP), and it is quasi-degenerate in mass with the dark matter lightest supersymmetric particle (LSP) neutralino (to within 10-20\% or so.) A very recent analysis [2] of this neutralino-stop cooannihilation scenario shows that the ATLAS search for supersymmetry [3], corresponding to an integrated luminosity of $1 \mathrm{fb}^{-1}$, essentially rules out an NLSP stop mass below $140 \mathrm{GeV}$. Future LHC searches will no doubt provide far more stringent constraints on this scenario, or perhaps discover neutralino-stop coannihilation. Another colored particle which can have mass of order $100 \mathrm{GeV}$ and can still survive the recent ATLAS bounds [4] is the sbottom quark when it is quasi-degenerate in mass with neutralino.

Motivated by these considerations we propose to investigate the neutralino-sbottom coannihilation scenario in this paper. The analysis in [1] shows that this scenario requires a framework larger than the CMSSM with additional soft supersymmetry breaking (SSB) parameters. We have selected to investigate neutralino-sbottom coannihilation in $S U(5)$, which naturally allows for the presence of additional parameters in accord with minimal supergravity [5]. We should note here that neutralino-sbottom coannihilation in $S U(5)$ has previously been explored in the references listed in [6]. Our analysis, we believe, provides for the first time a comprehensive study of this scenario in $S U(5)$. 
The outline for the rest of the paper is as follows. In section 2 we briefly describe the model, list the $S U(5)$ inspired SSB parameters, and the range of values employed in our scan. Section 3 describes the scanning procedure and the relevant experimental constraints that we have employed. The results pertaining to neutralino-sbottom coannihilation are discussed in section 4, and our conclusions are summarized in section 5 .

\section{Soft Supersymmetry Breaking (SSB) Parame- ters in $S U(5)$}

We will search for neutralino-sbottom coannihilation in the $S U(5)$ framework and, for simplicity, we assume that the SSB mass terms for sfermions are family independent. In $S U(5)$ the standard model (SM) fermions per family are allocated to the following representations: $\overline{5} \supset\left(d^{c}, L\right)$ and $10 \supset\left(Q, u^{c}, e^{c}\right)$, where in the brackets, we have employed standard notation for the SM fermions. It seems natural to consider two independent SSB scalar mass terms, at $M_{\mathrm{G}}, m_{\overline{5}}$ and $m_{10}$, for the matter multiplets. The MSSM Higgs doublets belong to $5\left(H_{u}\right)$ and $\overline{5}\left(H_{d}\right)$ representations of $S U(5)$, to which we assign two independent SSB mass terms, $m_{H_{u}}$ and $m_{H_{d}}$.

The minimal $S U(5)$ model predicts $b$ - $\tau$ Yukawa coupling unification at $M_{\mathrm{G}}$ from the interaction $\overline{5} \cdot 10 . \overline{5}_{H_{d}}$, and its LHC implications have been discussed in [1]. It was shown in [10] that $b-\tau$ Yukawa coupling unification can be relaxed by including either non-renormalizable interactions in the theory, or by employing a more complicated Higgs sector, beyond the minimal one. To implement neutralino-sbottom coannihilation, we find it helpful not to require $b-\tau$ Yukawa unification. We also invoke non-universal soft trilinear terms. In particular, we assume that at $M_{\mathrm{G}}$, there are two independent parameters $A_{b}\left(=A_{\tau}\right)$ and $A_{t}$, where the equality $A_{b}=A_{\tau}$ keeps the number of SSB parameters to a minimum. To summarize, we consider the following SSB terms for this study:

$$
M_{1 / 2}, m_{10}, m_{\overline{5}}, m_{H_{u}}, m_{H_{d}}, \tan \beta, A_{t}, A_{b}\left(=A_{\tau}\right) \text { and } \operatorname{sign}(\mu) .
$$

We shall see that this is the minimal set of independent MSSM terms which allows one to implement neutralino-sbottom coannihilation in $S U(5)$. We will set $\mu>0$ in this paper.

\section{Phenomenological constraints and scanning pro- cedure}

We employ the ISAJET 7.80 package [13] to perform random scans over the parameters listed in Eq.(1). In this package, the weak scale values of gauge and third 
generation Yukawa couplings are evolved to $M_{\mathrm{G}}$ via the MSSM renormalization group equations (RGEs) in the $\overline{D R}$ regularization scheme. We do not strictly enforce the unification condition $g_{3}=g_{1}=g_{2}$ at $M_{\mathrm{G}}$, since a few percent deviation from unification can be assigned to unknown GUT-scale threshold corrections [14]. The difference between $g_{1}\left(=g_{2}\right)$ and $g_{3}$ at $M_{G}$ is no worse than $4 \%$.

The various boundary conditions are imposed at $M_{\mathrm{G}}$ and all the SSB parameters, along with the gauge and Yukawa couplings, are evolved back to the weak scale $M_{\mathrm{Z}}$. In the evaluation of Yukawa couplings the SUSY threshold corrections [15] are taken into account at the common scale $M_{\mathrm{SUSY}}=\sqrt{m_{\tilde{t}_{L}} m_{\tilde{t}_{R}}}$, where $m_{\tilde{t}_{L}}$ and $m_{\tilde{t}_{R}}$ are the third generation left and right handed stop quarks. The entire parameter set is iteratively run between $M_{\mathrm{Z}}$ and $M_{\mathrm{G}}$ using the full 2-loop RGEs until a stable solution is obtained. To better account for leading-log corrections, one-loop step-beta functions are adopted for gauge and Yukawa couplings, and the SSB parameters $m_{i}$ are extracted from RGEs at multiple scales $m_{i}=m_{i}\left(m_{i}\right)$. The RGE-improved 1loop effective potential is minimized at an optimized scale $M_{\mathrm{SUSY}}$, which effectively accounts for the leading 2-loop corrections. Full 1-loop radiative corrections are incorporated for all sparticle masses.

The requirement of radiative electroweak symmetry breaking puts an important theoretical constraint on the parameter space. Another important constraint comes from limits on the cosmological abundance of stable charged particles [16]. This excludes regions in the parameter space where charged SUSY particles, such as $\tilde{\tau}_{1}$ or $\tilde{t}_{1}$, become the LSP. We keep only those solutions where the lightest neutralino is the LSP which, in most cases, saturates the WMAP (Wilkinson Microwave Anisotropy Probe) dark matter relic abundance bound. Neutralino-sbottom coannihilation plays an important role in realizing the desired LSP relic abundance.

We have performed random scans for the following parameter range:

$$
\begin{array}{r}
0 \leq m_{5}, m_{H_{d}} \leq 5 \mathrm{TeV} \\
0 \leq m_{10}, m_{H_{u}} \leq 10 \mathrm{TeV} \\
0 \leq M_{1 / 2} \leq 2 \mathrm{TeV} \\
-15 \mathrm{TeV} \leq \mathrm{A}_{\mathrm{t}} \leq 15 \mathrm{TeV} \\
-15 \mathrm{TeV} \leq \mathrm{A}_{\mathrm{b}}=\mathrm{A}_{\tau} \leq 30 \mathrm{TeV} \\
1.1 \leq \tan \beta \leq 60 \\
\mu>0
\end{array}
$$

where $m_{t}=173.3 \pm 1.1 \mathrm{GeV}$ [17] is the top quark pole mass. We use $m_{b}\left(m_{Z}\right)=2.83$ $\mathrm{GeV}$ which is hard-coded into ISAJET. The various boundary conditions in Eq.(2) are implemented through the scanning procedure. We found, for instance, that $m_{\overline{5}}$ has to be smaller than $m_{10}$ to realize the neutralino-sbottom coannihilation solutions.

In scanning the parameter space, we employ the Metropolis-Hastings algorithm 
as described in [18]. All of the collected data points satisfy the requirement of radiative electroweak symmetry breaking (REWSB), with the lightest neutralino in each case being the LSP. After collecting the data, we impose the mass bounds on all the particles [16] and use the IsaTools package [19] to implement the following phenomenological constraints on points that have sbottom coannihilation solution:

$$
\begin{aligned}
m_{h}(\text { lightest Higgs mass }) & \geq 114.4 \mathrm{GeV} \\
B R\left(B_{s} \rightarrow \mu^{+} \mu^{-}\right) & <1.1 \times 10^{-8} \\
2.85 \times 10^{-4} \leq B R(b \rightarrow s \gamma) & \leq 4.24 \times 10^{-4}(2 \sigma) \\
0.15 \leq \frac{B R\left(B_{u} \rightarrow \tau \nu_{\tau}\right)_{\mathrm{MSSM}}}{B R\left(B_{u} \rightarrow \tau \nu_{\tau}\right)_{\mathrm{SM}}} & \leq 2.41(3 \sigma) \\
\Omega_{\mathrm{CDM}} h^{2} & =0.111_{-0.037}^{+0.028}(5 \sigma)
\end{aligned}
$$

As far as the muon anomalous magnetic moment is concerned, we only require that the model does no worse than the SM.

\section{Sbottom Coannihilation}

To realize the desired LSP dark matter relic abundance through neutralino-sbottom coannihilation, the mass difference between these two should be less than or of order $20 \%$ [6]. In Figure 1 we present our results in the $M_{1 / 2}-m_{10} / m_{\overline{5}}, A_{t} / A_{b}-m_{10} / m_{\overline{5}}$, $A_{t} / A_{b}-m_{H_{u}} / m_{H_{d}}, m_{\overline{5}} / m_{H_{d}}-m_{H_{u}} / m_{H_{d}}$ planes, with the parameter values all defined at $M_{\mathrm{G}}$. Gray points are consistent with REWSB and LSP neutralino. The orange points satisfy, in addition, the particle mass bounds, constraints from $B R\left(B_{s} \rightarrow\right.$ $\left.\mu^{+} \mu^{-}\right), B R\left(B_{u} \rightarrow \tau \nu_{\tau}\right)$ and $B R(b \rightarrow s \gamma)$. The blue points form a subset of orange points and correspond to NLSP sbottom, but which is not closely degenerate in mass with the neutralino. The red points form a subset of blue points and represent neutralino-sbottom coannihilation, the scenario we are really after ! The vertical and the horizontal dashed lines show equality of parameters along the $\mathrm{x}$ and $\mathrm{y}$-axes respectively.

In order to explain our findings we consider the one loop renormalization group equations for the third generation squarks and sleptons [25]

$$
\begin{aligned}
16 \pi^{2} \frac{d}{d t} m_{\tilde{Q}_{3}}^{2} & =X_{t}+X_{b}-\frac{32}{3} g_{3}^{2}\left|M_{3}\right|^{2}-6 g_{2}^{2}\left|M_{2}\right|^{2}-\frac{2}{5} g_{1}^{2}\left|M_{1}\right|^{2}+\frac{1}{5} g_{1}^{2} S, \\
16 \pi^{2} \frac{d}{d t} m_{\tilde{u}_{3}^{c}}^{2} & =2 X_{t}-\frac{32}{3} g_{3}^{2}\left|M_{3}\right|^{2}-\frac{32}{15} g_{1}^{2}\left|M_{1}\right|^{2}-\frac{4}{5} g_{1}^{2} S \\
16 \pi^{2} \frac{d}{d t} m_{\tilde{d}_{3}^{c}}^{2} & =2 X_{b}-\frac{32}{3} g_{3}^{2}\left|M_{3}\right|^{2}-\frac{8}{15} g_{1}^{2}\left|M_{1}\right|^{2}+\frac{2}{5} g_{1}^{2} S \\
16 \pi^{2} \frac{d}{d t} m_{\tilde{L}_{3}}^{2} & =X_{\tau}-6 g_{2}^{2}\left|M_{2}\right|^{2}-\frac{6}{5} g_{1}^{2}\left|M_{1}\right|^{2}-\frac{3}{5} g_{1}^{2} S \\
16 \pi^{2} \frac{d}{d t} m_{\tilde{e}_{3}^{c}}^{2} & =2 X_{\tau}-\frac{24}{5} g_{1}^{2}\left|M_{1}\right|^{2}+\frac{6}{5} g_{1}^{2} S
\end{aligned}
$$


Here

$$
\begin{aligned}
X_{t} & =2\left|y_{t}\right|^{2}\left(m_{H_{u}}^{2}+m_{\tilde{Q}_{3}}^{2}+m_{\tilde{u}_{3}^{c}}^{2}\right)+2\left|A_{t}\right|^{2} \\
X_{b} & =2\left|y_{b}\right|^{2}\left(m_{H_{d}}^{2}+m_{\tilde{Q}_{3}}^{2}+m_{\tilde{d}_{3}^{c}}^{2}\right)+2\left|A_{b}\right|^{2} \\
X_{\tau} & =2\left|y_{\tau}\right|^{2}\left(m_{H_{d}}^{2}+m_{\tilde{L}_{3}}^{2}+m_{\tilde{e}_{3}^{c}}^{2}\right)+2\left|A_{\tau}\right|^{2} \\
S & \equiv \operatorname{Tr}\left[Y_{j} m_{\phi_{j}}^{2}\right]=m_{H_{u}}^{2}-m_{H_{d}}^{2}+\operatorname{Tr}\left[m_{\tilde{Q}}^{2}-m_{\tilde{L}}^{2}-2 m_{\tilde{u}^{c}}^{2}+m_{\tilde{d}^{c}}^{2}+m_{\tilde{e}^{c}}^{2}\right]
\end{aligned}
$$

and $\tilde{Q}_{3}, \tilde{u}_{3}^{c}, \tilde{d}_{3}^{c}, \tilde{L}_{3}, \tilde{e}_{3}^{c}$ denote the third generation squarks and sleptons. Also, $g_{i}$ and $M_{i}(i=1,2,3)$ denote the gauge couplings and gaugino masses for $U(1)_{Y}, S U(2)_{L}$ and $S U(3)_{C}$, and $y_{j}, A_{j}(j=t, b, \tau)$ are the third family Yukawa couplings and trilinear scalar SSB couplings, respectively.

In the $M_{1 / 2}-m_{10 /} m_{\overline{5}}$ plane of Figure 1, we show that in order to have sbottom NLSP, the ratio $m_{10} / m_{\overline{5}}$ should be greater than 1.4 (blue points). If we require successful neutralino-sbottom coannihilation, the ratio $m_{10} / m_{\overline{5}}>2$ (red points). In general, there is a two step process for realizing NLSP sbottom. First we consider the RGE effects, and in the second step we search for cancellation in the $2 \times 2$ sbottom mass matrix between the diagonal and off diagonal $\left(-m_{b}\left(A_{b}+\mu \tan \beta\right)\right)$ entries.

Let us see why it is not possible to have sbottom NLSP starting from universal sfermion masses $\left(m_{\overline{5}}=m_{10}\right)$. First, we note, that it is difficult to make the sbottom lighter than the stop. Eqs.(3)-(11) show that the gluino loop contribution raises the squark masses (we ignore the hypercharge contribution because it is sufficiently small), while the Yukawa and SSB trilinear couplings tend to lower them. The left handed squarks are heavier than the right handed ones due to the $S U(2)_{L}$ contribution. From the QCD point of view the sbottom and stop masses ${ }^{2}$ renormalize identically. To split them, the loop corrections involving Yukawa couplings must play a role. Since the top Yukawa coupling, for most $\tan \beta$ values, is larger than the bottom Yukawa coupling, it is hard to make $m_{\tilde{b}^{c}}^{2}<m_{\tilde{t}^{c}}^{2}$ through RG running. If the soft trilinear couplings $A_{t}$ and $A_{b}$ are independent of each other, one could make one of the sbottom mass eigenvalues lighter than the stop. But in this case we need to make sure that the eigenvalues of the stau mass ${ }^{2}$ matrix remain positive, after suitable cancellation in the sbottom mass ${ }^{2}$ matrix is accomplished. It turns out, as we show in $A_{t} / A_{b}-m_{10} / m_{\overline{5}}$ plane (Figure 1 ), that $m_{10}=m_{\overline{5}}$ and $A_{t}=A_{b}$ are not compatible with neutralino-sbottom coannihilation. It is possible to achieve neutralino-sbottom coannihilation with $A_{t}=A_{b}=A_{\tau}$, provided $m_{10} / m_{\overline{5}}>2$. The ratio $m_{10} / m_{\overline{5}}$ takes its minimal value around 1.4 , which corresponds to $A_{t} / A_{b}<-0.5$. Note that for $M_{1 / 2}>m_{0}$ where $m_{0}$ is the universal SSB mass term for sfermions at $M_{\mathrm{G}}$, it is difficult to make the sbottom lighter than the right handed stau because of QCD corrections from the gluino loop. Combining these two observations, it is clear that we need $m_{10}>m_{\overline{5}}$ for any given value of $M_{1 / 2}$.

In the $A_{t} / A_{b}-m_{H_{u}} / m_{H_{d}}$ plane in Figure 1 we see two viable neutralino-sbottom 

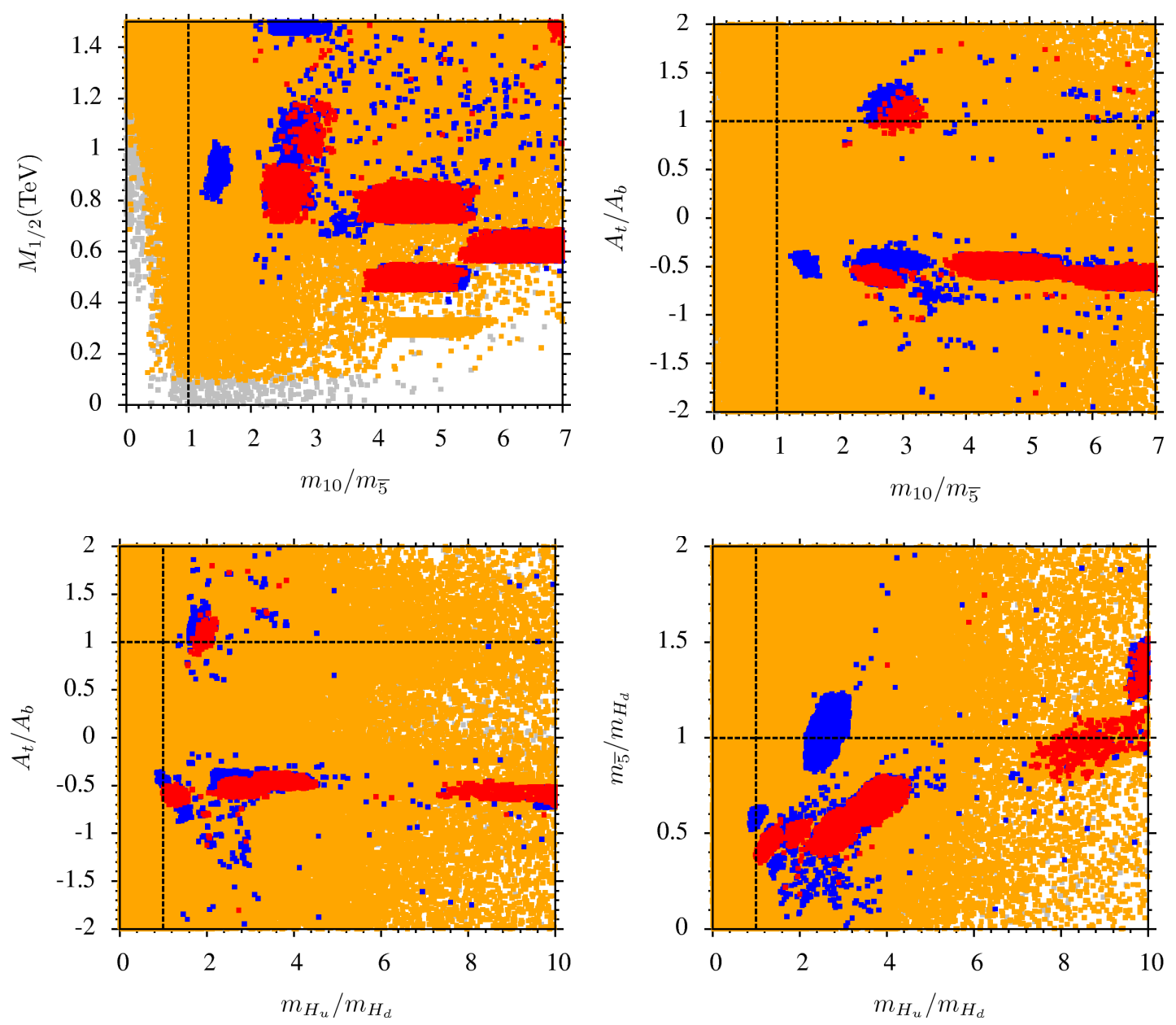

Figure 1: Plots in the $M_{1 / 2}-m_{10} / m_{\overline{5}}, A_{t} / A_{b}-m_{10} / m_{\overline{5}}, A_{t} / A_{b}-m_{H_{u}} / m_{H_{d}}, m_{\overline{5}} / m_{H_{d}}$ - $m_{H_{u}} / m_{H_{d}}$ planes. Gray points are consistent with REWSB, neutralino LSP. The orange points satisfy, in addition, the particle mass bounds, constraints from $B R\left(B_{s} \rightarrow \mu^{+} \mu^{-}\right), B R\left(B_{u} \rightarrow \tau \nu_{\tau}\right)$ and $B R(b \rightarrow s \gamma)$. The blue points form a subset of orange points that shows sbottom quark as an NLSP. The red points represent a subset of blue points that corresponds to the neutralino-sbottom coannihilation scenario. The vertical and horizontal dashed lines show equality of parameters along $\mathrm{x}$ and $\mathrm{y}$-axes respectively. 
coannihilation regions which have $A_{t} / A_{b}=1$ or $m_{H_{u}} / m_{H_{d}}=1$. However, we also see that it is not possible to have such solutions with both these ratios simultaneously equal to unity.

In minimal $S U(5)$ the MSSM down type Higgs field resides in the $\overline{5}$ representation, and so it is interesting to see whether or not neutralino-sbottom coannihilation or sbottom NLSP is possible by setting $m_{\overline{5}}=m_{H_{d}}$. In the $m_{\overline{5}} / m_{H_{d}}-m_{H_{u}} / m_{H_{d}}$ plane with $m_{\overline{5}}=m_{H_{d}}$, neutralino-sbottom coannihilation requires $m_{H_{u}} / m_{H_{d}} \geq 8$ (red points), while sbottom NLSP only needs $m_{H_{u}} / m_{H_{d}} \geq 2$ (blue points).

In Figure 2 we present results in the $m_{\overline{5}}-M_{1 / 2}, m_{\overline{5}}-m_{10}, A_{t}-A_{b}, m_{H_{d}}-m_{H_{u}}$, $\tan \beta-m_{\overline{5}}$ and $R_{b \tau}-\tan \beta$ planes which display the values of the SSB parameters required to yield sbottom NLSP/ coannihilation. Gray points are consistent with REWSB and neutralino LSP. The orange points satisfy, in addition, the particle mass bounds, constraints from $B R\left(B_{s} \rightarrow \mu^{+} \mu^{-}\right), B R\left(B_{u} \rightarrow \tau \nu_{\tau}\right)$ and $B R(b \rightarrow s \gamma)$. The blue points form a subset of orange points and correspond to sbottom NLSP. The red points represent a subset of the blue points and represent the neutralino-sbottom coannihilation scenario.

As we can see from the $m_{\overline{5}}-M_{1 / 2}$ panel, neutralino-sbottom coannihilation prefers relatively heavy gauginos $\left(M_{1 / 2}>400 \mathrm{GeV}\right)$, while $m_{\overline{5}}$ can be as light as $500 \mathrm{GeV}$. For $m_{\overline{5}}$ lighter than $500 \mathrm{GeV}$, it is difficult to separate the right handed sbottom mass from the left handed tau slepton. In the $m_{\overline{5}}-m_{10}$ plane, we see that $m_{10}$ should be larger than $2 \mathrm{TeV}$ in order to have neutralino-sbottom coannihilation.

As noted above, the RG running is not sufficient to realize an NLSP sbottom, and some cancellation is required in addition. Consider the plot in $A_{t}-A_{b}$ plane at SUSY scale where we see that neutralino-sbottom coannihilation solutions mostly require $\left|A_{b}\right|>\left|A_{t}\right|$, where $\left|A_{b}\right|$ can be $O(10-15) \mathrm{TeV}$. The off-diagonal entries $m_{b}\left(A_{b}+\mu \tan \beta\right)$ for the sbottom quark mass matrix are of comparable magnitude to the diagonal entries and, as a result, we can realize neutralino-sbottom coannihilation.

The plot in $m_{H_{d}}-m_{H_{u}}$ (at $M_{\mathrm{G}}$ ) plane shows that neutralino-sbottom coannihilation requires $m_{H_{u}}>m_{H_{d}}$. This can be understood by considering the one-loop RGEs for $m_{H_{u}}^{2}$ and $m_{H_{d}}^{2}$ :

$$
\begin{aligned}
16 \pi^{2} \frac{d}{d t} m_{H_{u}}^{2} & =3 X_{t}-6 g_{2}^{2}\left|M_{2}\right|^{2}-\frac{6}{5} g_{1}^{2}\left|M_{1}\right|^{2}+\frac{3}{5} g_{1}^{2} S \\
16 \pi^{2} \frac{d}{d t} m_{H_{d}}^{2} & =3 X_{b}+X_{\tau}-6 g_{2}^{2}\left|M_{2}\right|^{2}-\frac{6}{5} g_{1}^{2}\left|M_{1}\right|^{2}-\frac{3}{5} g_{1}^{2} S .
\end{aligned}
$$

Combining with Eqs. (8)-(10) we see that the RG evolution of $m_{H_{u}}^{2}$ and $m_{H_{d}}^{2}$ depend on $y_{t}, y_{b},\left|A_{b}\right|$ and $\left|A_{t}\right|$. Our conclusion from Figure 1 is that neutralino-sbottom coannihilation solutions require in most cases $\left|A_{t}\right|<\left|A_{b}\right|$, which means that $m_{H_{d}}^{2}$ renormalizes (decreases) more than $m_{H_{u}}^{2}$. In order to have $-m_{H_{d}}^{2}>-m_{H_{u}}^{2}$ at low scale, we need to start in most cases with $m_{H_{d}}^{2}<m_{H_{u}}^{2}$ at $M_{\mathrm{G}}$. 

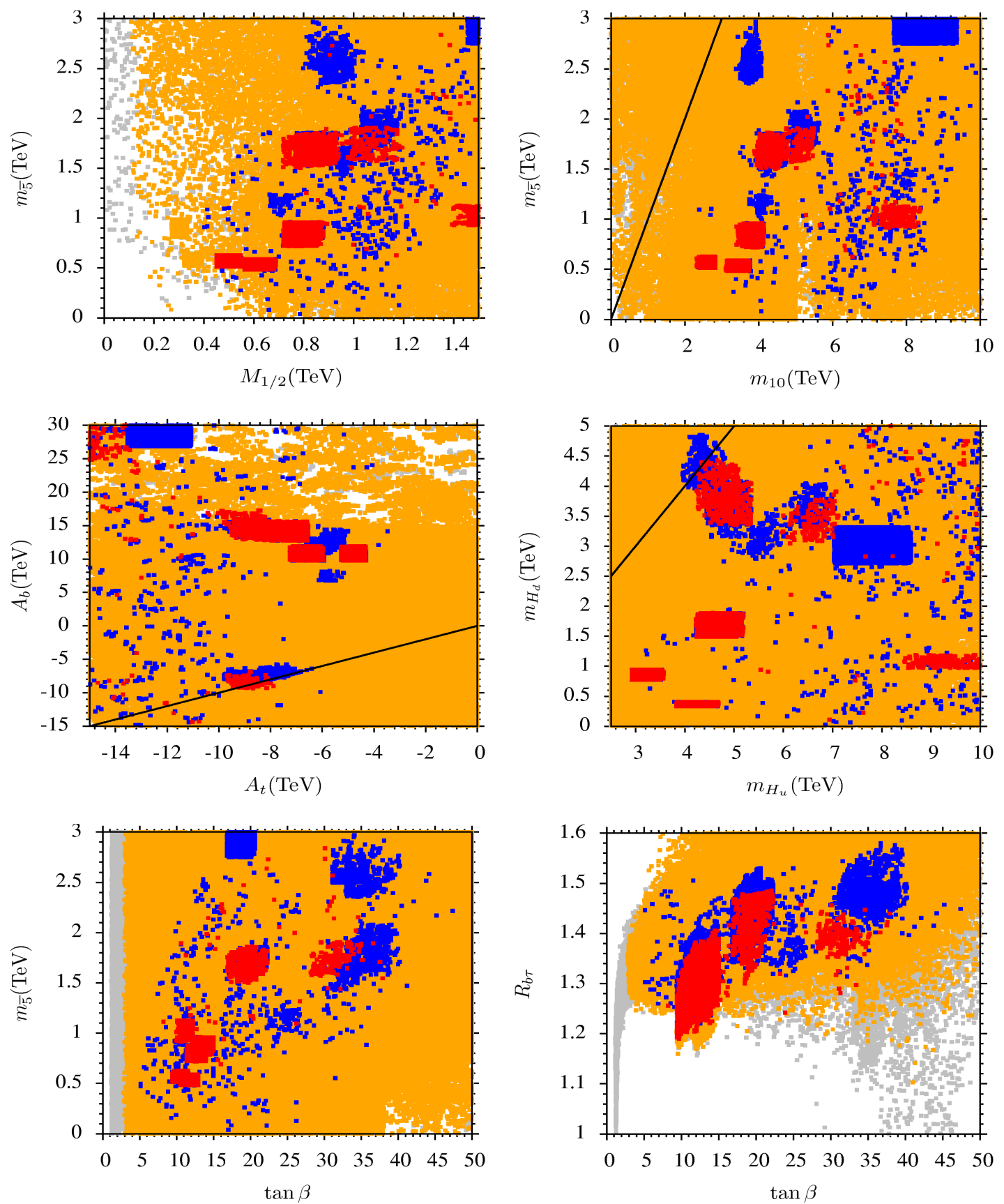

Figure 2: Plots in the $m_{1 / 2}-m_{\overline{5}}, m_{10}-m_{\overline{5}}, A_{t}-A_{b}, m_{H_{d}}-m_{H_{u}}, \tan \beta-m_{\overline{5}}$ and $R_{b \tau}-$ $\tan \beta$ planes. Color coding same as in Figure 1. Lines in black have unit slopes with $\mathrm{x}$ and $\mathrm{y}$ coordinates equal 
We note from $m_{\overline{5}}$ - $\tan \beta$ plane that sbottom coannihilation prefers small to moderately large values for $\tan \beta(7<\tan \beta<40)$. In this region the top Yukawa coupling reaches its minimum value at $M_{\mathrm{G}}$.

In order to quantify $b-\tau$ Yukawa unification, we define the quantity $R_{b \tau}$ as

$$
R_{b \tau}=\frac{\max \left(y_{b}, y_{\tau}\right)}{\min \left(y_{b}, y_{\tau}\right)}
$$

The $R_{b \tau}-\tan \beta$ plot in Figure 2 shows that it is difficult to reconcile $b-\tau$ Yukawa unification with the neutralino-sbottom coannihilation scenario. For the parameter range given in Eq.(2), $b-\tau$ Yukawa unification is at the level of $20 \%$ or so, which agrees with the results in ref. [10].

In Figure 3, we show plots in the $m_{\tilde{g}}-m_{\tilde{d}^{c}}, m_{\tilde{g}}-m_{\tilde{u}^{c}}, m_{\tilde{g}}-m_{\tilde{b}_{1}}, m_{\tilde{t}_{1}}-m_{\tilde{b}_{1}}, m_{\tilde{\tau}_{1}}-m_{\tilde{b}_{1}}$, and $m_{\tilde{t}_{1}}-m_{\tilde{\chi}_{1}^{0}}$, planes. The color coding is the same as in Figure 1, except that the green points in the $m_{\tilde{t}_{1}}-m_{\tilde{\chi}_{1}^{0}}$ plane form a subset of orange points and satisfy all current experimental bounds [3].

From the results recently presented by the ATLAS [3] and CMS [7] collaborations, our findings are just beginning to be tested. In the $m_{\tilde{d}^{c}}-m_{\tilde{g}}$ and $m_{\tilde{u}^{c}}-m_{\tilde{g}}$ planes we show graphs for the first generation squarks versus gluino mass. From the $m_{\tilde{d}^{c}}-m_{\tilde{g}}$ plane one sees that the minimum value of $m_{\tilde{d}^{c}}$ in the neutralino-sbottom coannihilation scenario is around $800 \mathrm{GeV}$, corresponding to a gluino mass of $1.2 \mathrm{TeV}$. From the plot in $m_{\tilde{u}^{c}}-m_{\tilde{g}}$ plane, we see that $m_{\tilde{u}^{c}}$ is heavy $(\gtrsim 2.2 \mathrm{TeV})$, and lies well above the current experimental bound.

The plot in $m_{\tilde{b}_{1}}-m_{\tilde{g}}$ plane shows that the neutralino-sbottom coannihilation solutions (red points) allow a relatively light sbottom quark, with mass as low as $210 \mathrm{GeV}$. The corresponding gluino mass is around $1.2 \mathrm{TeV}$. For gluino mass around $3 \mathrm{TeV}$, the corresponding value of $m_{\tilde{b}_{1}}$ in this scenario is around $700 \mathrm{GeV}$. The plot in $m_{\tilde{b}_{1}}$ - $m_{\tilde{t}_{1}}$ plane shows that in neutralino-sbottom coannihilation solutions, the minimum (maximum) value of the lighter stop quark mass is of order $300 \mathrm{GeV}(1.4 \mathrm{TeV}$.)

In Figure 3 we also show plots in $m_{\tilde{b}_{1}}-m_{\tilde{\tau}_{1}}$ and $m_{\tilde{b}_{1}}-m_{\tilde{\chi}_{1}^{0}}$ planes. In the $m_{\tilde{b}_{1}}-m_{\tilde{\chi}_{1}^{0}}$ plane, the green points represent solutions that satisfy all the constraint mentioned above. Points within $20 \%$ of the line with unit slope represent neutralino-sbottom coannihilation, while points in blue color represent just NLSP sbottom solutions (without coannihilation.) From the graph we see that the minimum value of neutralino mass in either case close to $210 \mathrm{GeV}$. The minimum value of stau mass corresponding to the coannihilation scenario is about $500 \mathrm{GeV}$.

In Figure 4 we show a plot in the $m_{\tilde{b}_{1}}-B R\left(B_{s} \rightarrow \mu^{+} \mu^{-}\right)$plane, with the color coding the same as in Fig. 1. The vertical dashed line on the left marks the SM prediction for $B R\left(B_{s} \rightarrow \mu^{+} \mu^{-}\right)$, while the dashed line on the right shows the current LHCd+CMS limit. As previously mentioned in section 3, we have applied the latest combined LHCd+CMS limit for $B R\left(B_{s} \rightarrow \mu^{+} \mu^{-}\right)$of $1.1 \times 10^{-8}$. This can be seen as a sharp cutoff in solutions represented by yellow points. We observe that the 

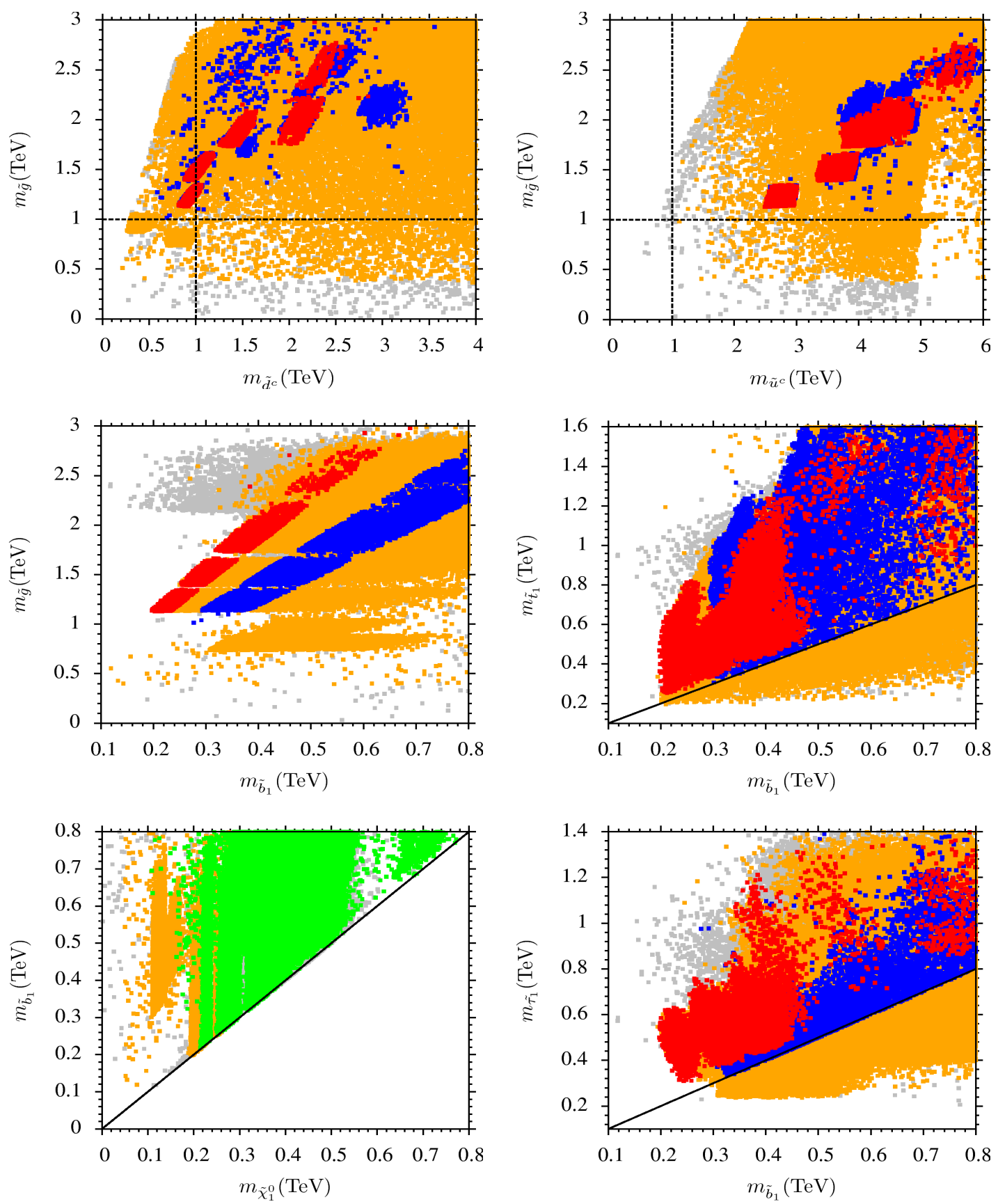

Figure 3: Plots in the $m_{\tilde{g}}-m_{\tilde{d}^{c}}, m_{\tilde{g}}-m_{\tilde{u}^{c}}, m_{\tilde{g}}-m_{\tilde{b}_{1}}, m_{\tilde{t}_{1}}-m_{\tilde{b}_{1}}$, and $m_{\tilde{\tau}_{1}}-m_{\tilde{b}_{1}}$ planes. Color coding is the same as in Figure 1 except that green points in $m_{\tilde{t}_{1}}-m_{\tilde{\chi}_{1}^{0}}$ plane form a subset of orange points which satisfies all current experimental bounds [3]. 


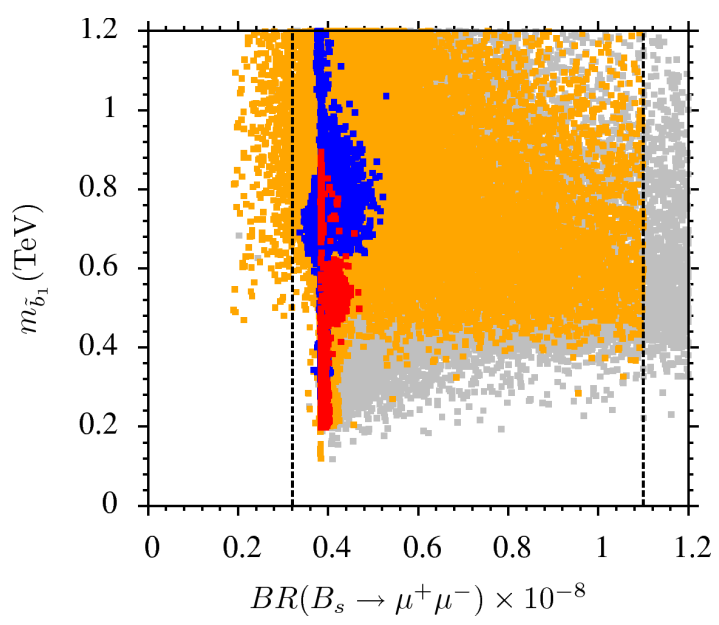

Figure 4: Plot in $m_{\tilde{b}_{1}}-B R\left(B_{s} \rightarrow \mu^{+} \mu^{-}\right)$plane. Color coding is the same as in Figure 1. Left vertical dashed line corresponds to the SM prediction for $B R\left(B_{s} \rightarrow \mu^{+} \mu^{-}\right)$ and right dashed line shows the current LHCd+CMS limit.

neutralino-sbottom coannihilation solutions predict a value for $B R\left(B_{s} \rightarrow \mu^{+} \mu^{-}\right)$of around $4 \times 10^{-9}$, close to the SM prediction of around $3.2 \times 10^{-9}$. This prediction of a slight excess in $B R\left(B_{s} \rightarrow \mu^{+} \mu^{-}\right)$for the neutralino-sbottom coannihilation scenario should be tested soon.

In Table 1 we present three characteristic benchmark points which satisfy all constraints presented in Chapter 3. Points 1 and 3 respectively represent the minimum and maximum values of the sbottom mass $(\sim 210 \mathrm{GeV}$ and $\sim 820 \mathrm{GeV})$, corresponding to neutralino-sbottom coannihilation. Point 2 represents just an NLSP sbottom solution (without insisting on correct dark matter relic abundance value), with the stop and stau masses relatively close to it. Since the LSP is essentially a pure bino, both its spin-independent $\left(\sim 10^{-13}-10^{-11} \mathrm{pb}\right)$ and spin-dependent $\left(\sim 10^{-10}-10^{-7} \mathrm{pb}\right)$ cross sections on nucleons are rather small [26]. Consequently it won't be easy to detect the LSP in direct and indirect experiments. However, as shown in [2] and [27], the LHC supersymmetry searches can be exploited to probe cross sections of this magnitude.

\section{Conclusions}

We have described in detail the conditions under which the neutralino-sbottom coannihilation scenario can be realized in supersymmetric $S U(5)$. In particular we have identified, for the first time we believe, the minimum number of soft supersymmetry breaking parameters that are required in order to have NLSP sbottom and neutralinosbottom coannihilation in $S U(5)$. The coannihilation scenario predicts the existence 


\begin{tabular}{lccc}
\hline & Point 1 & Point 2 & Point 3 \\
\hline$M_{1 / 2}$ & 453 & 591 & 1544 \\
$m_{10}$ & 2394 & 3626 & 7972 \\
$m_{5}$ & 519 & 577 & 926 \\
$\tan \beta$ & 10 & 12 & 10 \\
$A_{t}$ & -4347 & -7012 & -16000 \\
$A_{b}$ & 10080 & 9931 & 30010 \\
$m_{H_{d}}$ & 835 & 338 & 1121 \\
$m_{H_{u}}$ & 3065 & 4213 & 9116 \\
$s i g n(\mu)$ & + & + & + \\
\hline$m_{h}$ & 123 & 125 & 127 \\
$m_{H}$ & 536 & 1785 & 4251 \\
$m_{A}$ & 533 & 1773 & 4223 \\
$m_{H}$ & 541 & 1787 & 4252 \\
\hline$m_{\tilde{\chi}_{1,2}^{0}}$ & 194,374 & 260,505 & 703,1330 \\
$m_{\tilde{\chi}_{3,4}^{0}}$ & 822,831 & 2049,2051 & 4872,4873 \\
$m_{\tilde{\chi}_{1,2}^{ \pm}}$ & 374,840 & 505,2066 & 1332,4903 \\
$m_{\tilde{g}}$ & 1135 & 1460 & 3524 \\
\hline$m_{\tilde{u}_{L, R}}$ & 2546,2611 & 3785,3875 & 8429,8597 \\
$m_{\tilde{t}_{1,2}}$ & 372,1688 & 709,2584 & 1721,5743 \\
\hline$m_{\tilde{d}_{L, R}}$ & 2548,909 & 3786,1033 & 8430,2445 \\
$m_{\tilde{b}_{1,2}}$ & 213,1692 & 414,2610 & 823,5825 \\
\hline$m_{\tilde{\nu}_{1}}$ & 729 & 905 & 1840 \\
$m_{\tilde{\nu}_{3}}$ & 472 & 579 & 748 \\
\hline$m_{\tilde{e}_{L, R}}$ & 739,2308 & 926,3512 & 1890,7745 \\
$m_{\tilde{\tau}_{1,2}}$ & 499,2169 & 622,3371 & 917,7374 \\
\hline$\sigma_{S I}(\mathrm{pb})$ & $6.23 \times 10^{-11}$ & $7.61 \times 10^{-12}$ & $5.18 \times 10^{-13}$ \\
$\sigma_{S D}(\mathrm{pb})$ & $2.92 \times 10^{-7}$ & $1.87 \times 10^{-8}$ & $5.73 \times 10^{-10}$ \\
$\Omega_{C D M} h^{2}$ & 0.08 & 6.8 & 0.09 \\
\hline \hline & & & \\
\hline \hline & & & \\
\hline
\end{tabular}

Table 1: Point 1 shows the minimum value of sbottom mass $(213 \mathrm{GeV})$ for neutralinosbottom coannihilation. Point 2 represents a solution with sbottom NLSP $(414 \mathrm{GeV})$, more than 50\% heavier than the LSP neutralino. Point 3 represents a neutralinosbottom coannihilation solution with a heavier sbottom mass $(823 \mathrm{GeV})$.

of relatively heavy $(\gtrsim 1 \mathrm{TeV})$ gluino and first two generation squark masses. The NLSP sbottom in the coannihilation scenario is quasi-degenerate in mass with the LSP neutralino, and it can be as light as $210 \mathrm{GeV}$ or so, without running into conflict with the current supersymmetry search at the LHC. We also highlight a few benchmark points which can be tested at the LHC.

\section{Acknowledgments}

We thank M. Adeel Ajaib, T. Li, M. Papucci and O. Gonzalez Lopez for valuable discussions. This work is supported in part by the DOE Grant No. DE-FG02- 
91ER40626 (I.G., S.R. and Q.S.). This work used the Extreme Science and Engineering Discovery Environment (XSEDE), which is supported by the National Science Foundation grant number OCI-1053575.

\section{References}

[1] I. Gogoladze, S. Raza, Q. Shafi, arXiv:1104.3566 [hep-ph].

[2] M. Adeel Ajaib, T. Li, Q. Shafi, arXiv:1111.4467 [hep-ph].

[3] G. Aad et al. [ ATLAS Collaboration ], arXiv:1109.6572 [hep-ex].

[4] G. Aad et al. [ATLAS Collaboration], arXiv:1103.4344 [hep-ex].

[5] A. Chamseddine, R. Arnowitt and P. Nath, Phys. Rev. Lett. 49 (1982) 970; R. Barbieri, S. Ferrara and C. Savoy, Phys. Lett. B119 (1982) 343; N. Ohta, Prog. Theor. Phys. 70 (1983) 542; L. J. Hall, J. D. Lykken and S. Weinberg, Phys. Rev. D27 (1983) 2359; for a review see S. Weinberg, The Quantum Theory of Fields: Volume 3, Supersymmetry, Cambridge University Press (2000) 442p.

[6] S. Profumo, Phys. Rev. D68 (2003) 015006; C. Pallis, Nucl. Phys. B678 (2004) 398; A. Belyaev, T. Lastovicka, A. Nomerotski and G. Lastovicka-Medin, Phys. Rev. D81 (2010) 035011.

[7] S. Chatrchyan et al. [ CMS Collaboration ], arXiv:1109.2352 [hep-ex].

[8] T. Aaltonen et al. [ CDF Collaboration ], Phys. Rev. Lett. 105, 081802 (2010). [arXiv:1005.3600 [hep-ex]].

[9] K. Nakamura et al. [ Particle Data Group Collaboration ], J. Phys. G G37, 075021 (2010).

[10] I. Gogoladze, R. Khalid, N. Okada, Q. Shafi, Phys. Rev. D79, 095022 (2009).

[11] U. Chattopadhyay and P. Nath, Phys. Rev. D 65, 075009 (2002); S. Komine and M. Yamaguchi, Phys. Rev. D 65, 075013 (2002); S. Profumo, Phys. Rev. D 68, 015006 (2003); C. Pallis, Nucl. Phys. B 678, 398 (2004); C. Balazs and R. Dermisek, JHEP 0306, 024 (2003); W. Altmannshofer, D. Guadagnoli, S. Raby and D. M. Straub, Phys. Lett. B 668, 385 (2008); S. Antusch and M. Spinrath, Phys. Rev. D 79, 095004 (2009)

[12] M. Adeel Ajaib, T. Li, Q. Shafi, Phys. Lett. B701, 255 (2011).

[13] H. Baer, F. E. Paige, S. D. Protopopescu and X. Tata, arXiv:hep-ph/0001086. 
[14] J. Hisano, H. Murayama, and T. Yanagida, Nucl. Phys. B402 (1993) 46. Y. Yamada, Z. Phys. C60 (1993) 83; J. L. Chkareuli and I. G. Gogoladze, Phys. Rev. D 58, 055011 (1998).

[15] D. M. Pierce, J. A. Bagger, K. T. Matchev, and R.-j. Zhang, Nucl. Phys. B491 (1997) 3.

[16] K. Nakamura et al. [ Particle Data Group Collaboration ], J. Phys. G G37, 075021 (2010).

[17] [Tevatron Electroweak Working Group and CDF Collaboration and D0 Collab], arXiv:0903.2503 [hep-ex].

[18] G. Belanger, F. Boudjema, A. Pukhov and R. K. Singh, JHEP 0911, 026 (2009); H. Baer, S. Kraml, S. Sekmen and H. Summy, JHEP 0803, 056 (2008).

[19] H. Baer, C. Balazs, and A. Belyaev, JHEP 03 (2002) 042; H. Baer, C. Balazs, J. Ferrandis, and X. Tata Phys. Rev. D64 (2001) 035004.

[20] S. Schael et al. Eur. Phys. J. C 47, 547 (2006).

[21] $\mathbf{C M S}$ and $\mathbf{L H C b}$ Collaborations. "Search for the rare decay $B_{s, d} \rightarrow \mu^{+} \mu^{-}$at the LHC", LHCb-CONF-2011-047, CMS PAS BPH-11-019.

[22] E. Barberio et al. [Heavy Flavor Averaging Group (HFAG) Collaboration], arXiv:0704.3575 [hep-ex].

[23] E. Barberio et al. [Heavy Flavor Averaging Group], arXiv:0808.1297 [hep-ex].

[24] E. Komatsu et al. [WMAP Collaboration], Astrophys. J. Suppl. 180, 330 (2009).

[25] See for instance S. P. Martin, In *Kane, G.L. (ed.): Perspectives on supersymmetry* 1-98. [arXiv:hep-ph/9709356 [hep-ph]] and referances therein.

[26] I. Gogoladze, R. Khalid, Y. Mimura, Q. Shafi, Phys. Rev. D83, 095007 (2011).

[27] D. Feldman, Z. Liu and P. Nath, Phys. Rev. D 80, 015007 (2009) N. Chen, D. Feldman, Z. Liu, P. Nath and G. Peim, Phys. Rev. D 83, 035005 (2011) M. Adeel Ajaib, T. Li, Q. Shafi and K. Wang, JHEP 1101, 028 (2011) 